\title{
Früh sucht, wer die optimale Praxis finden will!
}

Ich werde öfter gefragt, wie man auf dem Weg in die Selbstständigkeit am besten an passende Praxisobjekte gelangt. Ein Jahr nach meiner Neugründung in Berlin kann ich dazu rückblickend sagen: Am besten schon im Studium die Fühler ausstrecken und Erfahrungen sammeln, damit man dann - wenn es darauf ankommt - weiß, was zu einem passt.

\section{Dr. Philipp F. Gebhardt //}

BISS45 - Kieferorthopädie Berlin

www.biss45.de

Dr. Gebhardt berichtet in jeder Ausgabe über seinen Weg vom abgeschlossenen Studium zum jungen Zahnarzt.

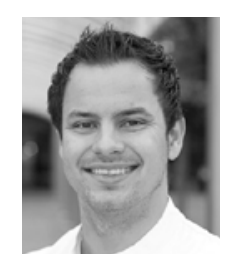

\section{$\mathrm{D}$}

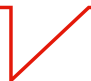
ie Frage, welcher Praxisstandort zu einem passt, ist sicherlich maßgeblich durch den jeweiligen Erfahrungsgrad geprägt, der direkt nach dem Studium natürlich noch nicht sehr ausgeprägt ist. Hierzu kann ich bestätigen, was ich schon vor einigen Jahren geschrieben habe: „Frühzeitig Erfahrungen zu sammeln und auch schon während des Studiums in Praxen zu hospitieren bzw. nach Städten zu suchen, die auch perspektivisch interessant sind, halte ich für einen guten Rat." Zwar hat man theoretisch nach dem Studium alle Zeit der Welt und das Durchschnittsalter für den Einstieg in die Selbstständigkeit liegt in der Zahnmedizin statistisch bei 36,7 Jahren. Allerdings zeigt eine Studie der Apotheker- und Ärztebank aus 2015 auch, dass die durchschnittlichen Investitionskosten bei unter 30-Jährigen bei 310.000 Euro, bei über 40-Jährigen jedoch etwa ein Drittel darunter liegen. Man könnte jetzt mutmaßen, dass ältere Kollegen aufgrund ihrer Erfahrung sinnvoller und deshalb weniger investieren. Ein möglicher Grund ist allerdings viel mehr, dass die jüngeren Kollegen in der Regel sowohl privat als auch beruflich weniger Kompromisse eingehen müssen und zudem einfach zehn Jahre mehr Zeit haben, die zusätzlichen Investitionen zu tilgen. Als Kompromisse sehe ich zum Beispiel Abstriche bei der Standortwahl sowie bei der Positionierung und Ausstattung der Praxis. Aber auch eine Minimierung der privaten Ausgaben, wie zum Beispiel der Miete, ist einige Jahre nach dem Studium einfacher als nach 15 Jahren Berufserfahrung.

Was das ideale Praxisobjekt angeht, finde ich es zielführend, möglichst früh exakte Vorstellungen zu entwickeln. Meiner Ansicht nach macht es auf jeden Fall Sinn, sich auf ein bis maximal zwei Regionen zu beschränken und in dieser Region auch schon mindestens ein paar Monate gelebt oder gearbeitet zu haben. Das verschafft einen nicht zu unterschätzenden Wettbewerbsvorteil. Sollte die Entscheidung dabei auf eine strukturstarke und überversorgte Region fallen, ist es fast unerlässlich, vor Ort schon Erfahrungen gesammelt zu haben. Denn ohne ein gutes Netzwerk dort ein ideales nachhaltiges Objekt zu finden, halte ich für ausgeschlossen.

\section{Impressum}

Eigentümer \& Copyright @ Springer-Verlag Berlin Heidelberg 2015, Springer Medizin c/o Springer-Verlag GmbH, Tiergartenstr. 17, 69121 Heidelberg, Tel. +49 6221/487-0 Springer Medizin ist ein Teil der Fachverlagsgruppe Springer Science+Business Media Geschäftsführung Springer Medizin:

Joachim Krieger (Managing Director Professional Group Springer Nature), Fabian Kaufmann

Director Apotheke und Zahnmedizin: Marion Bornemann Redaktionsleitung "der junge zahnarzt":

Dr. Swanett Koops (Tel. -8217, swanett.koops@springer.com) Redaktion CME-Rubrik: Dr. Swanett Koops (Tel. -8217, swanett.koops@springer.com) Layout: Arnulf Illing (Tel. -8952, arnulf.illing@springer.com)

Bildredaktion: Christiane Seufert

Anzeigenleitung: Marita Säuberlich (Tel. -8309, Fax -68309,

marita.saeuberlich@springer.com)

Corporate Publishing: Anja Weisse (Tel. -8739, Fax -68739, anja.weisse@springer.com)

Druck: PHOENIX PRINT GmbH, Würzburg. Printed in Germany

Erscheinungsweise: 4 Ausgaben pro Jahr

Papierausgabe: ISSN 1869-5744, gedruckt auf säurefreiem Papier

Bezugspreise (unverb. Preisempfehlung inkl. 7\% deutscher MwSt. und Versand): Vorzugspreis für persönliche Abonnenten: EUR 56,-- Einzelheftpreis: EUR 22,--. Das Abonnement kann jederzeit 2 Monate vor Ende des Bezugszeitraumes gekündigt werden. Bestellungen oder Rückfragen nimmt jede Buchhandlung oder der Verlag entgegen.
Kontakt: Springer Customer Service Center GmbH, Haberstr. 7, 69126 Heidelberg, Tel. +49 6221/345-4303, Fax -4229, Leserservice@springer.com (Mo.-Fr. 8.00 Uhr bis 20.00 Uhr) Copyright \& allgemeine Hinweise: Die Zeitschrift sowie alle in ihr enthaltenen einzelnen Beiträge und Abbildungen sind urheberrechtlich geschützt. Jede Verwertung, die nicht ausdrücklich vom Urheberrechtsgesetz zugelassen ist, bedarf der vorherigen schriftlichen Zustimmung des Verlags. Das gilt insbesondere für Vervielfältigungen, Bearbeitungen, Übersetzungen, Mikroverfilmungen und die Einspeicherung und Verarbeitung in elektronischen Systemen.

Gezeichnete Artikel geben nicht unbedingt die Meinung der Redaktion wieder. Autoren können unter bestimmten Voraussetzungen an der Ausschüttung der Bibliotheks- und Fotokopietantiemen teilnehmen. Einzelheiten bei VG WORT, Abt. Wissenschaft, Goethestr. 49, 80336 München.

Angaben über Dosierungsanweisungen und Applikationsformen sind anhand anderer Literaturstellen oder der Packungsbeilage auf ihre Richtigkeit zu überprüfen. Der Verlag übernimmt keine Gewähr.

Der IVW (Informationsgemeinschaft zur Feststellung der Verbreitung von Werbeträgern) angeschlossen. 